\title{
Foot Complications in Patients with Diabetes: Experience in a Teaching Hospital, Rajshahi, Bangladesh
}

\author{
AKM Shamsul Haque ${ }^{1, ~ *, ~ H a b i b u n ~ N o b i ~ M d ~ S h a f i q u z z a m a n ~}{ }^{2}$, Md Shohorab Hossain ${ }^{1}$, \\ Goutom Kumar Ghosh ${ }^{3}$ \\ ${ }^{1}$ Department of Surgery, Pabna Medical College, Pabna, Bangladesh \\ ${ }^{2}$ Department of Surgery, Rajshahi Medical College, Rajshahi, Bangladesh \\ ${ }^{3} 250$ Bedded General Hospital, Pabna, Bangladesh
}

\section{Email address:}

drmasud71@gmail.com (AKM S. Haque)

${ }^{*}$ Corresponding author

\section{To cite this article:}

AKM Shamsul Haque, Habibun Nobi Md Shafiquzzaman, Md Shohorab Hossain, Goutom Kumar Ghosh. Foot Complications in Patients with Diabetes: Experience in a Teaching Hospital, Rajshahi, Bangladesh. Journal of Surgery. Vol. 8, No. 6, 2020, pp. $171-177$. doi: $10.11648 /$ j.js.20200806.11

Received: June 16, 2020; Accepted: July 13, 2020; Published: November 4, 2020

\begin{abstract}
Diabetic foot complications are the most common cause of non-traumatic lower extremity amputations. The aim of this study was to determine the different pattern of foot complication associated with Diabetes mellitus. Methods \& Materials: This experimental study was conducted on a total of 50 patients of diabetic foot complications admitted in the department of Surgery (in-patient) in Rajshahi Medical College \& Hospital (RMCH), Rajshahi, Bangladesh during the period from June 2009 to December, 2009. Either sex ranging from above 15 years were included in the study. Approval committee of BCPS approved the dissertation protocol including the Ethical clearance. Written consent was obtained from each subject. Data were collected using a structured questionnaire containing all the variables of interest (research instrument). The researcher used simple statistical data analyzed tools to analyze the collected data to determine the findings of this study. Results: A total of 50 diabetic patients were included in this study as per inclusion criteria of either sex. Among the patients $30(60 \%)$ were male and $20(40 \%)$ were female. The age group of the patients (30-40) years, were $02(4 \%),(41-50)$ years, were $10(20 \%),(51-$ $60)$ years, were $32(64 \%),(61-70)$ years, were $06(12 \%)$. The overall diabetic foot complications of the patients were as follows: ulceration were $13(26 \%)$ cellulitis were $08(16 \%)$, cellulitis with abscess were $21(42 \%)$, gangrene were $06(12 \%)$, gangrene with septicemia was $01(2 \%)$, and bony involvement was $01(2 \%)$. The trauma were found in $28(56 \%)$ patients. After operation, the foot complication prevailed in $06(12 \%)$ patients and from skin diseases, the foot complication arose in 04 $(08 \%)$ patients as well as from other causes $12(24 \%)$ patients suffered from foot complications. Neurological changes were noted only in $12(24 \%)$ patients. Vascular changes were found only in the feet of the $06(12 \%)$ patients. Mid foot were the highest number $30(60 \%)$ of the location of foot ulcers. Culture was done $25(50 \%)$ of the studied patients. Proteus were 05 $(20 \%)$, Klebsiela were $03(12 \%)$, Staphylococci were $04(16 \%)$ and Pseudomonus were $02(8 \%)$. Pain impaired/absent in one/both feet were found in $31(62 \%)$ of the patients. Impaired/absent temperature sensation was noted in $07(14 \%)$ of the patients and absent ankle reflex were noted in 09 (18\%) patients. Dry skin were 14 (28\%), hyper pigmentation were $05(10 \%)$, hypo pigmentation were $04(8 \%)$, cracked skin were $06(12 \%)$ and normal skin was found in 21 (42\%) cases. Wound debridement and dressing were 23 (46\%) highest in treatment modalities option. Conclusion: Combined with an aggressive wound care program, can result in a favorable outcomes.
\end{abstract}

Keywords: Peripheral Neuropathy, Diabetes Mellitus, Neurological Changes, Foot Ulcers, Bacteriological Study

\section{Introduction}

Diabetic foot complications are the most common cause of non-traumatic lower extremity amputations. The risk of lower extremity amputation is 15 to 46 times higher in diabetes than in persons who do not have diabetes mellitus 
[1]. Furthermore foot complications are the most frequent reason for hospitalization in patients with diabetes [2]. The vast majority of diabetic foot complications resulting in amputation begin with the formation of skin ulcers. Early detection and appropriate management of these ulcers may prevent up to 85 percent of amputations [3]. Unfortunately several studies [4] have found that primary care physicians infrequently perform foot examinations in diabetic patients during routine office visits. The feet of hospitalized diabetic patients may also be inadequately evaluated [5]. Peripheral arterial occlusive disease is four times more prevalent in diabetics than in non-diabetics [6]. The arterial occlusion typically involves the tibial and peroneal arteries but spares the dorsal ped artery [7]. Smoking, hypertension and hyperlipidemia commonly contributes the prevalence of peripheral arterial occlusive diseases in diabetics [8] Peripheral arterial disease is observed more frequently in patients of diabetes as compared with age-matched controls. Lower limb atherosclerosis in the diabetic patient is characterized by preferential involvement of the lower leg, in $70 \%$ of diabetic patients compared with only $20 \%$ of nondiabetic patients. Involvement of the deep femoral artery is also typical of diabetes. Other abnormality that is seen with diabetes is stenosis of multiple arterial segments. The risk factors predisposing to macroangiopathy and ischemic foot lesions are the same as the risk factors in non-diabetic patients, particularly smoking. The diabetic patient is also at an excess risk a due to constellation of risk factors especially uncontrolled hyperglycemia. Distal symmetric polyneuropathy is perhaps the most common complication affecting the lower extremities of patients with diabetes mellitus. This complication occur in up to 58 percent of patients with long standing disease [9]. Neuropathy, a major etiologic component of most diabetic foot ulcerations, is present in more than 82 percent of diabetic patients with foot wounds. This lack of protective sensation, combined with unaccommodated foot deformities, exposes patients to undue sudden or repetitive stress that leads to eventual ulcer formation with a risk of infection and possible amputation. Foot deformities, which are common in diabetic patients, leads to focal areas of high pressure. When an abnormal focus of pressure is coupled with lack of sensation, a foot ulcer can develop. Most diabetic foot ulcers form over areas of bony prominence, especially when bunions, calluses or hammer-toe formation lead to abnormally prominent bony points. Foot deformities are believed to be more common in diabetic patients due to atrophy of the intrinsic musculature responsible for stabilizing the toes [10]. A diabetic patient with a history of previous ulceration or amputation is an increased risk for further ulceration, infection and subsequent amputation. Alteration in foot dynamics due to ulceration, joint deformity or amputation can cause the abnormal distribution of planter pressure and result in formation in new ulcers [11]. Meticulous attention to foot care and proper management of minor foot injuries are key to preventing ulcer formation. Daily foot inspection by the patient (or a caretaker if the patient lacks sufficient visual acuity or mobility to perform the examination) is the cornerstone of proper foot care. Despite the best intentions and careful attention to foot care, many diabetic patients eventually develop foot ulcers. These wounds are principal portal of entry for infection in patients with diabetes. Frequently, the ulcers are covered by callus or fibrotic tissue. This makes the trimming of hyperkeratotic tissue important for comprehensive wound evaluation. Because these ulcers almost always form in patients with neuropathy, they are typically painless. Adequate debridement is the first step in the evaluation of foot ulcer. Debridement should remove all necrotic tissue and surrounding callus until a healthy bleeding edge is revealed. Patients (and physicians) often underestimate the need for debridement and may be surprised by appearance of the newly debrided ulcer. Recognition of risk factors, preventive foot maintenance and regular foot examinations are essential in preventing foot complications in patients with diabetes. When foot ulcers develop despite preventive measures, a systematically applied regimen of diagnosis and classification, coupled with early and appropriate treatment, should help to reduce tremendous personal and social burden of diabetes related amputations. However, there are very few studies and limited data regarding foot complications in patients with diabetes in Bangladesh. So, the researcher purposively designed this study. The aim of this study was to determine the different pattern of foot complication associated with Diabetes mellitus.

\section{Objectives}

\section{General Objectives}

To assess the different patterns of foot complications associated with Diabetes mellitus.

Specific Objectives

a) to assess peripheral neuropathy associated with diabetic foot.

b) to assess associated peripheral vascular disease.

c) to assess diabetic foot ulcers.

\section{Methodology and Materials}

This experimental study was conducted on a total of 50 patients of diabetic foot complications admitted in the department of Surgery (in-patient) in Rajshahi Medical College \& Hospital (RMCH), Rajshahi, Bangladesh during the period from June 2009 to December, 2009. Approval committee of BCPS approved the dissertation protocol including the Ethical clearance. Keeping compliance with Helsinki Declaration for Medical Research Involving Human Subjects 1964, all patients were informed verbally about the study design and right for the participant to withdraw from the project at any time, for any reason, what so ever. Written consent was obtained from each subject. Data were collected using a structured questionnaire containing all the variables of interest (research instrument). The researcher used simple statistical data analyzed tools to analyze the collected data to determine the findings of this study. The inclusion and exclusion criteria of the participants were as follows: 


\section{Inclusion criteria}

Those cases were included in the study, who fulfilled the following criteria:

1) Willing and able to participate after the study has been explained.

2) Older than the age of legal consent.

3) History of Diabetes mellitus

4) Foot complications with varying severity

Exclusion criteria:

1) Unwilling to give informed consent to take part in the study.

2) Patients less than 15 years of old.

3) Patients with acute medical emergency.

\section{Results}

A total of 50 diabetic patients were included in this study as per inclusion criteria of either sex. Among the patients 30 $(60 \%)$ were male and $20(40 \%)$ were female. The age group of the patients $(30-40)$ years, were $02(4 \%),(41-50)$ years, were $10(20 \%),(51-60)$ years, were 32 (64\%), (61-70) years, were $06(12 \%)$ The maximum incidence was observed in the 5 th and 6th decades of life. Occupation of the patients, trader were $27(54 \%)$, civil servant were $14(28 \%)$, unemployed were $09(18 \%)$. Educational background of the patients, no academic educational background were $08(16 \%)$, primary were $22(44 \%)$, secondary were $14(28 \%)$, graduation and above were $06(12 \%)$. Smoking habit of the male patients, smoker or ever smoked were $27(90 \%)$, non-smoker were 03 $(10 \%)$. Literacy about preventive measures of diabetic of the patients, have diabetic education from diabetic clinic were 21 (42\%), have no diabetic education from diabetic clinic were $29(58 \%) .18(36 \%)$ patients had adequate knowledge on foot care. On the other hand, $32(64 \%)$ patents had inadequate knowledge on foot care. $36(72 \%)$ patients had appropriate knowledge on footwear and 14 (28\%) patients had inappropriate knowledge on footwear. $24(48 \%)$ patients had good knowledge on foot hygiene and $26(52 \%)$ patients had poor knowledge on foot hygiene. On admission, the blood sugar level of the patients, $<7$ was $01(2 \%),(7-10)$ were 04 (8\%), (11-20) were $42(84 \%)$, and $>20$ were $03(6 \%)$. Duration of diabetes mellitus before onset of diabetic foot, $<5$ years were $04(8 \%),(6-10)$ years were $10(20 \%),>10$ years were $28(56 \%)$, newly detected were $08(16 \%)$. On admission blood sugar situation was found controlled 02 (4\%) and uncontrolled were $48(96 \%)$. Blood pressure of the patients was found normotensive, $39(78 \%)$ and hypertensive 11 (22\%). Normal lipid profile noted in $45(90 \%)$ patients and hyperlipidemia was found in $05(10 \%)$ patients. S.creatinine/ Blood sugar was within normal limit in $42(84 \%)$ patients and above normal range was found in $08(16 \%)$ patients. The mode of onset of diabetic foot of the patients, ulceration were $13(26 \%)$ cellulitis were $08(16 \%)$, cellulitis with abscess were $21(42 \%)$, gangrene were $06(12 \%)$, gangrene with septicemia was $01(2 \%)$, and bony involvement was $01(2 \%)$. The diabetic foot complication, trauma were found in 28 $(56 \%)$ patients, of them, accidental were $10(20 \%)$, nail cutting were $08(16 \%)$, ill-fitting shoes were $06(12 \%)$, thermal were $02(4 \%)$ and others were $02(4 \%)$. After operation, the foot complication prevailed in $06(12 \%)$ patients and from skin diseases the foot complication arose in $04(08 \%)$ patients as well as from other causes $12(24 \%)$ patients suffered from foot complications. Among the feet of 38 (76\%) patients no neurological changes were found. Neurological changes were noted only in $12(24 \%)$ patients, of them, sensory were $06(12 \%)$, motor were $02(4 \%)$, autonomic were $02(4 \%)$, and combination were 02 (4\%). No vascular changes were found in the feet of the $44(88 \%)$ diabetic patients but vascular changes were found only in the feet of the $06(12 \%)$ patients. Location of foot ulcers: dorsal toes were $09(18 \%)$, planter toes, fore foot and mid foot were $30(60 \%)$, heel $07(14 \%)$, dorsal foot were $03(6 \%)$, and multiple areas of the foot was $01(2 \%)$. Bacteriological study of wound swab: culture was not done of the $25(50 \%)$ patients. Culture was done $25(50 \%)$ of the studied patients, of them, in $05(20 \%)$ in the wound swab of the patients, no bacterial growth was found, E coli was found in the wound swab of 06 (24\%) patients, Proteus were 05 (20\%), Klebsiela were $03(12 \%)$, Staphylococci were $04 \quad(16 \%)$ and Pseudomonus were $02(8 \%)$. Presentations of peripheral neuropathy: Pain impaired/absent in one/both feet were found in $31(62 \%)$ of the patients. Impaired/absent temperature sensation was noted in $07(14 \%)$ of the patients and absent ankle reflex were noted in $09(18 \%)$ patients. Skin changes associated with diabetic foot: Dry skin were 14 (28\%), hyper pigmentation were 05 (10\%), hypo pigmentation were $04(8 \%)$, cracked skin were $06(12 \%)$ and normal skin was found in $21(42 \%)$ cases. Treatment modalities of diabetic foot: Wound debridement and dressing were $23(46 \%)$, skin graft was 07 (14\%), secondary closure was $09(18 \%)$, incision, drainage and dressing were $07(14 \%)$ and amputation was found in $04(8 \%)$ cases.

Table 1. Age \& Sex distribution of the studied patients $(n=50)$.

\begin{tabular}{lll}
\hline Age group (years) & $\mathbf{N}$ & $\%$ \\
\hline $30-40$ & 2 & 4 \\
$41-50$ & 10 & 20 \\
$51-60$ & 32 & 64 \\
$61-70$ & 6 & 12 \\
Male & 30 & 60 \\
Female & 20 & 40 \\
\hline
\end{tabular}

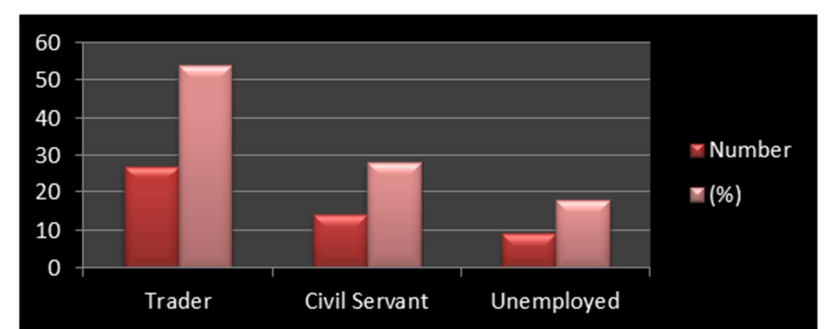

Figure 1. Occupation of the studied patients $(n=50)$. 
Table 2. Educational background of the studied patients $(n=50)$.

\begin{tabular}{lll}
\hline Level of education & N & \% \\
\hline No educational background & 8 & 16 \\
Primary & 22 & 44 \\
Secondary & 14 & 28 \\
Graduation \& above & 6 & 12 \\
\hline
\end{tabular}

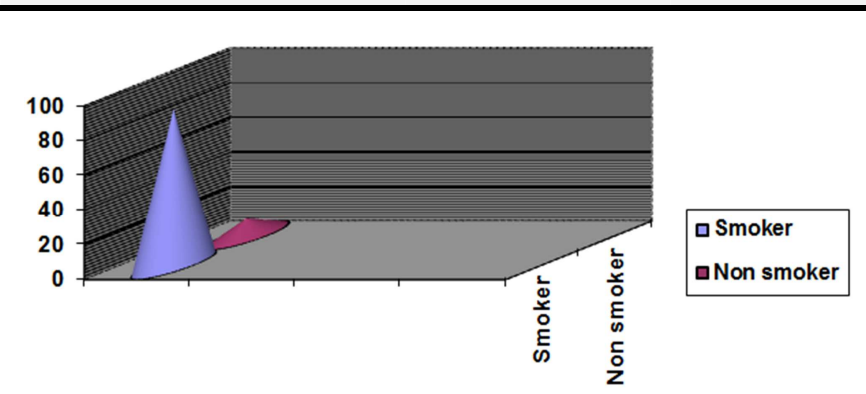

Figure 2. Smoking habit among male patients $(n=30)$.

Table 3. Literacy about preventive measures $(n=50)$.

\begin{tabular}{llll}
\hline Type of knowledge & & N & $\mathbf{\%}$ \\
\hline Education from & Have diabetic education & 21 & 42 \\
diabetic clinic & No diabetic education & 29 & 58 \\
foot care knowledge & Adequate & 18 & 36 \\
Knowledge of & Inadequate & 32 & 64 \\
footwear & Appropriate knowledge & 36 & 72 \\
Knowledge of foot & Good knowledge & 14 & 28 \\
hygiene & Poor knowledge & 26 & 48 \\
\hline
\end{tabular}

Table 4. On admission blood sugar level of the patients $(n=50)$.

\begin{tabular}{lll}
\hline Random Blood sugar & N & \% \\
\hline$<7$ & 1 & 2 \\
$7-10$ & 4 & 8 \\
$11-20$ & 42 & 84 \\
$>20$ & 3 & 6 \\
\hline
\end{tabular}

Table 5. Duration of diabetes mellitus before onset of diabetic foot $(n=50)$.

\begin{tabular}{lll}
\hline Duration of diabetes in years & $\mathbf{N}$ & $\mathbf{\%}$ \\
\hline$<5$ & 4 & 8 \\
$6-10$ & 10 & 20 \\
$>10$ & 28 & 56 \\
Newly detected & 8 & 16 \\
\hline
\end{tabular}

Table 6. Situation of blood sugar level on admission period $(n=50)$.

\begin{tabular}{lll}
\hline Blood sugar level $(\mathbf{m m o l} / \mathbf{L})$ & Controlled & Uncontrolled \\
\hline Number of patients & 2 & 48 \\
Percentage (\%) & 4 & 96 \\
\hline
\end{tabular}

Table 7. Blood pressure status of the studied patients $(n=50)$.

\begin{tabular}{lll}
\hline Blood pressure & N & \% \\
\hline Normotensive & 39 & 78 \\
Hypertensive & 11 & 22 \\
\hline
\end{tabular}

Table 8. Lipid profile of the studied patients $(n=50)$.

\begin{tabular}{lll}
\hline Lip ID Profile & N & \% \\
\hline Normal & 45 & 90 \\
Hyperlipidemia & 5 & 10 \\
\hline
\end{tabular}

Table 9. Renal status of the studied patients $(n=50)$.

\begin{tabular}{lll}
\hline S.creatinine/ Blood sugar & N & $\%$ \\
\hline Within normal limit & 42 & 84 \\
Above normal range & 8 & 16 \\
\hline
\end{tabular}

Table 10. Mode of onset of diabetic foot $(n=50)$.

\begin{tabular}{lll}
\hline Presentation & N & \% \\
\hline Ulceration & 13 & 26 \\
Cellulitis & 8 & 16 \\
Cellulitis with abscess & 21 & 42 \\
Gangrene & 6 & 12 \\
Gangrene with septicemia & 1 & 2 \\
Bony involvement & 1 & 2 \\
\hline
\end{tabular}

Table 11. Presentation of diabetic foot complications $(n=50)$.

\begin{tabular}{llll}
\hline No. & Mode & N & \% \\
\hline & Trauma & 28 & 56 \\
& Accidental & 10 & 20 \\
1 & Nail cutting & 8 & 16 \\
& Ill-fitting shoes & 6 & 12 \\
& Thermal & 2 & 4 \\
& Others & 2 & 4 \\
3 & After operation & 6 & 12 \\
4 & Skin diseases & 4 & 8 \\
\hline
\end{tabular}

Table 12. Neurological changes of the foot $(n=50)$.

\begin{tabular}{lll}
\hline Changes & N & \% \\
\hline No Changes & 38 & 76 \\
Neurological changes & 12 & 24 \\
Sensory & 6 & 12 \\
Motor & 2 & 4 \\
Autonomic & 2 & 4 \\
Combination & 2 & 4 \\
\hline
\end{tabular}

Table 13. Diabetic foot with vascular changes (50).

\begin{tabular}{lll}
\hline Changes & N & \% \\
\hline No changes & 44 & 88 \\
Vascular changes & 6 & 12 \\
\hline
\end{tabular}

Table 14. Location of foot ulcers $(n=50)$.

\begin{tabular}{lll}
\hline Site & N & \% \\
\hline Dorsal toes & 9 & 18 \\
Planter toes, fore foot and mid foot & 30 & 60 \\
Heel & 7 & 14 \\
Dorsal foot & 3 & 6 \\
Multiple areas of the foot & 1 & 2 \\
\hline
\end{tabular}

Table 15. Bacteriological study of wound swab $(n=50)$.

\begin{tabular}{lllll}
\hline \multicolumn{2}{c}{ Culture not done } & & $\mathbf{2 5}$ & $\mathbf{5 0 \%}$ \\
\hline & No growth & & 5 & $20 \%$ \\
Culture & & E coli & 6 & $24 \%$ \\
done & & Proteus & 5 & $20 \%$ \\
$(50 \%$ & Growth & Klebsiela & 3 & $12 \%$ \\
cases) & & Staphylococci & 4 & $16 \%$ \\
& & Pseudomonus & 2 & $8 \%$ \\
\hline
\end{tabular}


Table 16. Presentations of peripheral neuropathy $(n=50)$.

\begin{tabular}{lll}
\hline Presentations & N & \% \\
\hline Pain impaired/absent in one/both feet & 31 & 62 \\
Impaired/absent temperature sensation & 7 & 14 \\
Absent ankle reflex & 9 & 18 \\
\hline
\end{tabular}

Table 17. Skin changes associated with diabetic foot $(n=50)$.

\begin{tabular}{lll}
\hline Type of changes & N & \% \\
\hline Dry skin & 14 & 28 \\
Hyper pigmentation & 5 & 10 \\
Hypo pigmentation & 4 & 8 \\
Cracked skin & 6 & 12 \\
Normal & 21 & 42 \\
\hline
\end{tabular}

Table 18. Treatment modalities of diabetic foot $(n=50)$.

\begin{tabular}{lll}
\hline Treatment & N & \% \\
\hline Wound debridement and dressing & 23 & 46 \\
Skin graft & 7 & 14 \\
Secondary closure & 9 & 18 \\
Incision, drainage and dressing & 7 & 14 \\
Amputation & 4 & 8 \\
\hline
\end{tabular}

\section{Discussion}

Complications associated with diabetic foot ulcerations and the related co-morbid conditions are becoming a pandemic. With the worldwide number of diabetic patients reaching well into the hundreds of millions, the impact and burden on healthcare systems is felt on a global scale. Ten million people in the US were known to have diabetes in 1993, of which $15 \%$ had diabetic foot problems [12]. The prevalence of severe diabetic foot complications has been estimated at 10\% in Stockholm, Sweden [13]. Diabetic foot complications are experienced worldwide and affect both sexes. Diabetes mellitus is a complex metabolic disorder that affects 1-2 percent of the population. It can give rise to many tissue complications, among which, foot is particularly vulnerable to circulatory and neurological disorder, so that even minor trauma can lead to ulceration and infection. Careful observation and assessment of the wound is essential to ensure that the limb is not threatened, which could result in amputation. A careful multidisciplinary team approach is the key to the successful management of diabetic foot complications [14]. This study revealed a myriad of foot complications among people with diabetes attending admitting in surgery department, the most common being the diabetic foot ulcer. Most other foot complications were being managed by the dermatology unit of the hospital. People with fungal infection, onycholysis, yellow nails, colour changes, cracked skin and dry skin responded to dermatological treatment. However, these foot complications are still high in frequency compared with figures from the UK and the US [13]. Twenty one (42\%) of this study group had diabetes education from our diabetes clinic and this was reflected in the number of individuals who had adequate knowledge of foot care 18 (36\%), appropriate knowledge of foot wear $36(72 \%)$ and good knowledge of foot hygiene 24 (48\%). When the literacy level of this study group was assessed it was still very far from that of developed countries. Our study showed that 8 $(16 \%)$ of individuals did not have any background education, while $22(44 \%)$ and $14(28 \%)$ patients had primary and secondary education, respectively and rest 6 (12\%) patients had graduation or above degree. Social status could be another important factor in this sub-region. Twenty-seven (54\%) individuals were traders (mainly petty traders), 14 (28\%) were civil servants and $9(18 \%)$ were unemployed or students. If it was not for the diabetes education that is given to these individuals whenever they attend the clinic, the prevalence of diabetic foot complications in our society would be much higher. This shows that we still have a lot of work to do to improve the care and education for people with diabetes. Diabetic foot problems to be more common in men than women, especially in uncontrolled diabetes, with plasma glucose levels exceeding $7.8 \mathrm{mmol} / 1$ and a duration of diabetes exceeding 10 years [15]. In the present study the mean FBG was $8.68 \mathrm{mmol} / 1$, while $56 \%$ patient suffering from diabetes for more than 10 years. Out of 50 cases $30(60 \%)$ male and $20(40 \%)$ female patients suffering from diabetic foot with different types of presentations, Dr. Kalivani [16] agreed with these findings of this present study. In his study on the title "Evaluation of Diabetic Foot Complications According to Amit Jain's Classification", the male-female same ratio was prevailed as we found in our study. Eight patients presented with diabetic foot complication were detected as diabetic at ward and they did not know previously that they were suffering from diabetes. Others were known of their diabetes and a number of them could give the history of nature of their diabetic control. The range of blood glucose of admitted patient vary from $<7 \mathrm{mmol} / 1$ to more than 20 $\mathrm{mmol} / \mathrm{l}$. 42 patients $(84 \%)$ out of $50 \mathrm{had}$ their blood glucose level within $11-20 \mathrm{mmol} / \mathrm{l}$. only $3(6 \%)$ patients had their blood glucose level above $20 \mathrm{mmol} / \mathrm{l}$. Only 5 out of 50 patients had controlled blood sugar level, remaining 45 patients had uncontrolled sugar level. Some of the patients were on oral hypoglycemic drugs and some others were taking insulin. Out of this study population, 13 people had a diabetic foot ulcer $(26 \%), 8(16 \%)$ patients presented with cellulites, 21 patients $(42 \%)$ presented with cellulites with abscess, $6(12 \%)$ with gangrene, $1(2 \%)$ with gangrene with septicemia and $1(2 \%)$ with bony involvement. In our series we observed that $56 \%$ of the patient developed foot complications after trauma and among these group 20\% suffered foot complications after an accidental trauma, 16\% from nail cutting, $12 \%$ from ill-fitting shoe, $4 \%$ from thermal injury and rest $4 \%$ from other mode of trauma. Twelve percent of the patient developed foot complication after an surgical intervention and rest of the patients mode of onset of complication were from a diverse aetiology agreed Khalid et al [17] with these findings of our study. Thirty nine out of 50 patients were found normotensive and 11 patients were hypertensive. Patients who are genetically predisposed to the development of hypertension may be especially at risk. Among the 30 male patients 27 were 
either smoker or ever smoked before. Neurological changes were found in 12 patients. Six patients were suffered from sensory impairment. 2 from motor dysfunction, 2 with autonomic and rest 2 patients had a combination of above. Six out of 50 patients had vascular changes. Patients who were suffering from peripheral neuropathy presented with impaired/ absent pain of different degree and either impaired or absent temperature sensation. In these group we found that $31(62 \%)$ number of patients presented with either impaired or absent pain sensation and 7 (14\%) with impaired or absent temperature sensation. While performing ankle reflex test we find $9(18 \%)$ had no ankle reflex, almost the similar result was found in the study of Frykberg et al [18]. In our study culture from wound swab was done in 25 cases. Among them $5(10 \%)$ patients had no growth, 6 $(12 \%)$ had E.coli, $5(10 \%)$ with Proteus species, $3(6 \%)$ had Klebsiela, 4 (8\%) with Staphylococci and rest 2 (4\%) were found to have Pseudomous growth. Among the patients who suffered from foot ulcers maximum number $(60 \%)$ had their ulcer in Planter toes, fore foot and mid foot. Rest had ulcers in different location and according to the findings they were in dorsal toes $(18 \%)$, heel $(14 \%)$, dorsal foot $(6 \%)$ and multiple areas of foot $(2 \%)$, these findings were supported by Eneroth et al [19]. Five percent of the patient had Hyperlipidemia, 16\% had serum creatinine level above normal. Patients presented with different types of skin lesions. Out of 50 patients 14 (28\%) patients had dry skin, 5 $(10 \%)$ presented with hyper pigmentation, 4 (8\%) with hypo pigmentation, $6(12 \%)$ had cracked skin and rest 21 patient were devoid of any skin lesions. Varieties of surgical procedures were performed and these were attempted to limit the spread of infection and in severe cases amputation were performed. Abscesses were found in 21 cases and they were treated by incision, drainage of the pus and dressing. This procedure was done under local anesthesia and sometimes without any anesthesia. A broad spectrum antibiotic was instituted to prevent further spread of infection and to control hyperglycemia, fever and leucocytosis. After availability of culture report antibiotic coverage been narrowed as much as possible [20]. As with abscesses, broad spectrum antibiotic was used until bacteriology was determined. As wound became granulating and healthy, either secondary closure $(18 \%$ cases) or split thickness skin graft (14\%) was done. Twelve percent patients developed gangrene in the affected area. Amputation was done in $4(8 \%)$ patients for feet gangrene. Two patients had toe amputation, 1 bellow knee and rest 1 patient treated by above knee amputation, supported by Reiber et al [21].

\section{Limitations of the Study}

This was an experimental study with a limited sample size and small area. This study was conducted over a limited period of time. Only the entitled patient's got opportunity participated in this study. So, the limited sample size, short duration, and a limited study area were the limitations of this study. So, with a large sample, vast area and large span of time, the study may be further conducted.

\section{Conclusion and Recommendations}

Although a multidisciplinary "team" approach to the diabetic foot has been demonstrated as an effective treatment model and should be employed where feasible, a close working relationship with a patient's PCP is always a priority. This, when combined with an aggressive wound care program, can result in a favorable outcomes. The wound healing team must maintain an added level of diligence in their care and monitor for any and all indications of diabetic foot complications. The importance of foot care should be emphasized when diabetes is first diagnosed and reinforced at regular intervals. However, in those who develop ulcers, further re-education is needed to try and prevent another ulcer. In particular, neuropathic ulceration should be preventable with good foot care and appropriate footwear. The use of walking plasters has been advocated by some people. For instance, plaster casts and the 'Scotch cast' boot70 have both been used to aid ulcer healing as they enable the individual to remain mobile, but remove pressure from the ulcer site. Such casts and boot are not readily available in our country but alternative footwear that does not put persistent pressure on the ulcer can be improvised by indigenous manufacturers to serve the same purpose. Clubs and associations for people with diabetes and their relatives (supported by health workers concerned with their treatments) will allow them to discuss their problems, educate each other and pool their resources.

\section{References}

[1] Lavery LA, Ashry HR, vann Houtum W, Pugh JA, Harkless LB, Basu S. Variation in the incidence and proportion of diabetes related amputations in minorities. Diabetes care 1996; 19: 48-52.

[2] Gibbons G., Eliopoulos GM. Infection of diabetic foot. In: Kozak GP, et al., eds. Management of diabetic foot problems. Philadelphia: Saunders, 1984: 97-102.

[3] United States National Diabetes Advisory Board. The national long-range plan to combat diabetes. Bethesda, Md.: U. S. Department of Health and Human Services, Public Health Service, National Institute of Health, 1987: NIH publication number 88-1587.

[4] Wylie-Rosset J., Walker EA, Shamoon H., Engel S., BAsch C., Zybert P. Assessment of documented foot examinations for patients with diabetes in inner-city primary care clinics. Arch Fam Med 1995; 4: 46-50.

[5] Edelson GW, Armstrong DG, Lavery LA, Caicco G. The acutely infected diabetic foot is not adequately evaluated in an inpatient setting. Arch Intern Med 1996; 156: 2373-8.

[6] Kannel WB, McGee DL, Diabetes and glucose tolerance as risk factors for cardiovascular disease: the Framingham study. Diabetes care 1979; 2: 120-6.

[7] LoGerfo FW, Coffman JD. Vascular and microvascular disease of the foot in diabetes. Implications for foot care. $\mathrm{N}$ Engl J. Med 1984; 311: 1615-9. 
[8] Lee JS, Lu M., Lee VS, Russell D., Bahr C., Lee ET. Lower extremity amputation. Incidence, risk factors and mortality in the Oklahoma Indian Diabetes Study. Diabetes 1993; 42: 87682 .

[9] Harati Y. Diabetic peripheral neuropathy. In: Kominsky SJ, ed. Medical and surgical management of the diabetic foot. St. Louis: Mosby, 1994: 73-85.

[10] Brand PW. The insensitive foot (including leprosy). In: Jahss $\mathrm{MH}$, ed. Disorders of the foot \& ankle: medical and surgical management. 2d ed. Philadelphia: Saunders, 1991: 2173-5.

[11] Bild DE, Selby JV, Sinnock P., Browner WS, Braveman P., Showstack JA. Lower-extremity amputation in people with diabetes. Epidemiology and preventation. Diabetes Care 1989; 12: $24-31$

[12] Kinghton OR, Fiegel VD (1993) Growth factors and comprehensive Surgical care of Diabetic Wounds. Current Opinion in General Surgery 30: 32-39.

[13] Rosenquist U. (1982) An epidemiological survey of diabetic foot problems in the Stockholm country. Acta Medica Scandinavica 687: 55-60.

[14] Medica Scandinavica 687: 55-6025. International Working Group on the Diabetic Foot. International Consensus on the Diabetic Foot, Amsterdam, The Netherlands, 1999.
[15] Humphrey AP, Dowse GK, Thoma K., Zimmet PZ (1996) Diabetes and non-traumatic lower limb extremity amputations. Incidence, risk factors and prevention - a 12year follow up study in Nauru. Diabetes Care 19: 710-714.

[16] Kalivani V., Evaluation of Diabetic Foot Complications According to Amit Jain's Classification", J. Clin Dign Res. 2014, Dec, 8 (12): NC07-NC09.

[17] Khalid et al, "Diabetic Foot Complications and Their Risk Factors from a Large Retrospective Cohort Study", POLOS ONE>v. 10 (5), 2015, PMC: 44226557.

[18] Frykberg, R. G., Lavery, L., Pham, H., Harvey, C., Harkless, L., Veves, A. Role of neuropathy and high foot pressures in diabetic foot ulceration. Diabetes Care 21: 1714-1719, 1998.

[19] Eneroth, M., Apelqvist, J., Stenstrom, A. Clinical characteristics and outcome in 223 diabetic patients with deep foot infections. Foot Ankle Int 18: 716-722, 1997.

[20] Ha Van, G., Siney, H., Danan, J. -P., Sachon, C., Grimaldi, A. Treatment of osteomyelitis in the diabetic foot: contribution of conservative surgery. Diabetes Care 19: 1257-1260, 1996.

[21] Reiber, G. E., Boyko, E. J., Smith, D. G. Lower extremity foot ulcers and amputations in diabetes. In: Diabetes in America, 2nd ed. (NIH publ. no. 95-1468), edited by M. I. Harris, C. Cowie, and M. P. Stern, U. S. Government Printing Office, Washington, DC, 1995. 